\title{
Adaptation and mislocalization fields for saccadic outward adaptation in humans
}

\author{
Fabian Schnier \\ Dept. Psychology and Otto Creutzfeld Center for Cognitive and Behavioral Neuroscience, \\ Westfälische Wilhelms-Universität Münster, Germany \\ Eckart Zimmermann \\ Dept. Psychology and Otto Creutzfeld Center for \\ Cognitive and Behavioral Neuroscience, \\ Markus Lappe \\ Dept. Psychology and Otto Creutzfeld Center for \\ Cognitive and Behavioral Neuroscience,
}

Westfälische Wilhelms-Universität Münster, Germany Westfälische Wilhelms-Universität Münster, Germany

\begin{abstract}
Adaptive shortening of a saccade influences the metrics of other saccades within a spatial window around the adapted target. Within this adaptation field visual stimuli presented before an adapted saccade are mislocalized in proportion to the change of the saccade metric. We investigated the saccadic adaptation field and associated localization changes for saccade lengthening, or outward adaptation. We measured the adaptation field for two different saccade adaptations (14 deg to 20 $\mathrm{deg}$ and $20 \mathrm{deg}$ to $26 \mathrm{deg}$ ) by testing transfer to 34 different target positions. We measured localization judgements by asking subjects to localize a probe flashed before saccade onset. The amount of adaptation transfer differed for different target locations. It increased with increases of the horizontal component of the saccade and remained largely constant with deviation of the vertical component of the saccade. Mislocalization of probes inside the adaptation field was correlated with the amount of adaptation of saccades to the probe location. These findings are consistent with the assumption that oculomotor space and perceptual space are linked to each other.
\end{abstract}

Keywords: Saccade, outward adaptation, adaptation field, motor space, localization, perceptual space

\section{Introduction}

We perceive our environment with goal-directed movements that bring the fovea with high resolution to the region of interest. These gaze shifts are called saccades. Performing a saccade requires the conjoined action of sensory stages and motor stages in the central nervous system. The sensory input that is generated by the saccade target has to be transformed into motor commands which activate the eye muscles to perform the saccade towards the target. These oculomotor transformations have to guarantee for the appropriate saccade execution. If a saccade permanently fails to reach the target the corresponding transformation parameters will be adjusted. The ability to compensate for such continuing visual error is called saccadic adaptation. Because visual feedback is too slow to control

Corresponding author: Fabian.Schnier@uni-muenster.de M. L. is supported by the German Science Foundation DFG LA-952/3 and DFG LA-952/4, the German Federal Ministry of Education and Research project Visuo-Spatial Cognition, and the EC Project Eyeshots. saccadic eye movements during flight, stages of sensorimotor transformations have to be involved in these adaptive processes. It is not fully clear which brain regions and which corresponding signals are responsible for the adaptation (Hopp \& Fuchs, 2004; Pelisson, Alahyane, Panouilleres, \& Tilikete, 2010).

To investigate the phenomena of saccadic adaptation and its consequences one has to induce a consistent mismatch of computed saccade command and retinal feedback signal. This has been done in the monkey by weakening the horizontal recti muscles of one eye (Optican \& Robinson, 1980). Non-invasive adaptive experiments on humans and monkeys work with the so called McLaughlin Paradigm (McLaughlin, 1967). In these experiments an intrasaccadic target step results in the essential visual error or mismatch to induce saccadic adaptation. To reach effective adaptation the mismatch information should be provided immediately after the saccade (Shafer, Noto, \& Fuchs, 2000). Because no corrective saccades are needed to provide saccadic adaptation (Wallman \& Fuchs, 1998), the visual error itself must be the most important source for the inducement to adapt (Noto \& Robinson, 2001).

Adaptation with the McLaughlin paradigm is much 
faster than adaptation in the physiological or lesion studies. In monkeys typical trial counts for a saturated adaptation level in visually guided saccades are of about 1500 while in humans a steady state level can be already reached within 100 trials (Hopp \& Fuchs, 2004). Therefore this type of simulated adaptation is also called short term saccadic adaptation.

It is well known that the saccadic adjustments are direction specific (Deubel, Wolf, \& Hauske, 1986) and amplitude dependent (Albano \& King, 1989). They transfer to a bounded sector around the saccade target but not to orthogonal or oppositely directed vectors (Deubel, 1987; Semmlow, Gauthier, \& Vercher, 1989; Noto, Watanabe, \& Fuchs, 1999; Alahyane, Devauchelle, Salemme, \& Pelisson, 2008). The amount of transfer depends on the metric difference to the adapted saccade (Albano, 1996; Frens \& Van Opstal, 1994). Measuring all transfers from the adapted saccade to other saccades leads to a spatial region in which every coordinate is directly linked to the amount of transferred adaptation. This spatial window is called the adaptation field. The adaptation field was previously measured in monkeys for inward and outward adaptation (Noto et al., 1999) and in humans for inward adaptation (Collins, Dore-Mazars, \& Lappe, 2007). These measurements revealed an asymmetric transfer along the adapted axis but a relatively symmetric transfer along the orthogonal axis. A comparison with oculomotor movement fields in the superior colliculus and the frontal eye field pointed out a remarkable similarity leading to the assumption that those areas may be involved in the adaptation process (Noto et al., 1999).

Although the adaptation fields in saccadic shortening and lengthening in the monkey seemed to be rather similar, differences in the adaptation process itself between the two directions have been found (Noto et al., 1999; Straube, Fuchs, Usher, \& Robinson, 1997; Cecala \& Freedman, 2009). For example, many more saccades were required to produce adequate gain increases in saccadic outward adaptation than to produce the same gain decrease in saccadic inward adaptation (Straube et al., 1997; Scudder, Batourina, \& Tunder, 1998; Robinson, Noto, \& Bevans, 2003). Moreover, the state of an outward adapted monkey seemed to be more instable than the state of the corresponding inward adapted monkey, leading to a faster deadaptation in the outward adapted monkey (Straube et al., 1997). These results suggest that saccadic outward and inward adaptation could be based upon mechanisms with different dynamics.

Differences between inward and outward adaptation have also been found in a number of human studies (Miller, Anstis, \& Templeton, 1981; Semmlow et al., 1989; Deubel, 1991; Straube \& Deubel, 1995; Ethier, Zee, \& Shadmehr, 2008; Panouilleres et al., 2008). In one study, patients with lesions in the cerebellar vermis were tested for their ability to adapt outward and inward in the McLaughlin paradigm (Golla et al., 2008). These patients were able to adapt inward with a drop in saccade velocity and the corresponding duration decrease. However these patients were not able to increase saccadic amplitude during outward adaptation. The difference had to do with the patients disability to prolong the duration of a saccade. Measuring saccadic performance throughout many trials of the same amplitude (resilience experiment) yielded a drop in saccadic peak velocity that was not compensated for by an increase in saccade duration. Considering the similarity between inward adaptation and this resilience experiment the authors suggested that inward adaptation is a process that has substantial passive components of fatigue after many identical trials, and that saccadic outward adaptation is an active process that requires selective increases in saccade duration.

These results were in good accordance with Ethier et al. (2008) who compared the velocity profiles of adapted saccades with those of unadapted saccades of the same amplitude in relation to an optimal control model of saccade generation (Chen-Harris, Joiner, Ethier, Zee, \& Shadmehr, 2008; Ethier et al., 2008; Xu-Wilson, ChenHarris, Zee, \& Shadmehr, 2009). As a result of their comparison Ethier et al. (2008) suggested that in inward adaptation the internal feedback has to be adjusted midflight during the saccade while in outward adaptation the brain learned to produce larger saccade amplitudes by target remapping.

Indeed there are a neurophysiological (Catz, Dicke, \& Thier, 2008) and behavioral studies which would support the above mentioned inward and outward adaptation differences and indicate that both are based upon different mechanisms. The behavioral studies focused on inward and outward adaptation transfer to different saccade types (e.g. anti-saccades), hand pointing, and perception. Panouilleres et al. (2008) revealed a significant transfer of prosaccades to antisaccades performed in the adapted direction after inward adaptation but not after outward adaptation. Hernandez et al. (2008) found changes in hand pointing direction after outward adaptation but not after inward adaptation (Hernandez, Levitan, Banks, \& Schor, 2008). Zimmermann and Lappe (2010) showed that saccadic adaptation induced perceptual mislocalization even during subsequent fixation periods, but more so during outward than during inward adaptation (Zimmermann \& Lappe, 2010). Also studies that investigated spatial adaptation transfer patterns in humans for saccadic inward and outward adaptation documented a different transfer pattern between saccadic inward and outward adaptation (Miller et al., 1981; Semmlow et al., 1989). For saccadic inward adaptation the humans adaptation field has already been investigated (Collins et al., 2007) but because of differences between inward and outward adaptation a thorough investigation also in the outward adaptation is required. The first goal of our study is, therefore, the measurement of the 2D outward 
adaptation field in humans.

The second goal of our study is the measurement of the spatial pattern of perceptual localization changes induced by saccadic outward adaptation. Saccadic adaptation is known to affect the perceptual localizations of objects presented before an adapted saccade (Bahcall \& Kowler, 1999; Awater, Burr, Lappe, Morrone, \& Goldberg, 2005; Bruno \& Morrone, 2007; Georg \& Lappe, 2009; Zimmermann \& Lappe, 2009). Probes which were presented more than $100 \mathrm{~ms}$ before saccade onset revealed an adaptation-induced shift which was always in the direction of the adaptation.

Collins et al. (2007) investigated the adaptationinduced shift within a large spatial area surrounding the saccade target and compared it to the adaptation field for inward adaptation. They used three different localization test conditions. In the target on and target off conditions subjects had to perform a rightward saccade from a fixation point towards a 12 deg eccentric target that stepped 4 deg inward during the saccade. About $100 \mathrm{~ms}$ before saccade onset a flash appeared at one of 38 testing positions, and subjects had to localize the flash with a mouse pointer. In the target on condition the saccade target was visible as a post-saccadic visual reference. In the target off condition the target was extinguished after saccade performance. In a third, fixation, condition subjects had to suppress the saccade, fixate continuously, and localize the flash from the corner of their eyes.

Collins et al. (2007) found in the target on as well as in the target off condition mislocalization around the saccade target in the inward direction. The distribution of mislocalization was asymmetric in the horizontal direction and symmetric in the vertical direction. In the horizontal direction mislocalization increased with increasing distance from fixation point. In the vertical direction mislocalization decreased with deviation from the adapted saccade. The overall mislocalization in the target on condition was stronger than in the target off condition, suggesting that the post-saccadic visual reference led to an additional localization component in inward direction. The mislocalization field in the target on condition seemed to be parallel, while the target off mislocalization field was more radially directed towards fixation point. No significant inward shifts were found in the fixation condition. Comparison between the inward adaptation field and the target off mislocalization field revealed fundamental similarities. Therefore, Collins et al. (2007) suggested a link between perceptual space as measured by localization and motor space as measured by the adaptation field.

A connection between motor space and perceptual space might exist in saccadic outward adaptation too, but remains unexplored up to now. On the other hand, because recent studies have shown fundamental differences between inward and outward adaptation, adaptation field characteristics and the corresponding mislocalization fields could be different in the two cases.
To clarify this question, we investigated the saccadic adaptation field and the associated mislocalization for saccade lengthening or outward adaptation. We measured the adaptation field by testing transfer to 34 different target positions with different amplitudes and directions in complete darkness (field saccade condition). Localization judgements were recorded by asking the subjects to localize a probe flashed before saccade onset with a mouse pointer. We tested the same localization conditions as used in the experiment for saccadic inward adaptation (Collins et al., 2007). First, the target on localization condition, where the saccade target was visible throughout the whole saccadic performance and after the saccade. Second, the target off localization condition, where the saccade target was extinguished during saccadic performance and could not be used as a post-saccadic visual reference. Third, the fixation localization condition, where subjects were asked to fixate at the fixation point without performing a saccade and localize the probes which were flashed after a short fixation period.

\section{Methods}

\section{Participants}

Six subjects (4 females, 2 males) took part in all experiments. One subject was the first author. The other subjects were students at the university. All of them had normal or corrected to normal vision. Their age ranged from 22 to 28 years. Subjects had participated in some other eye movement sessions before the experiment. All participants gave informed consent in accordance with the Declaration of Helsinki.

\section{Experimental setup}

Experiments were done on a Macintosh PC for stimulus presentation. The stimulus monitor was a 21" (55 $\mathrm{cm})$ Eizo FlexScan F930 with a vertical frequency of $120 \mathrm{~Hz}$ at a resolution of $1024 \times 768$ pixels, resulting in a visual field of $40 \mathrm{deg} \times 30 \mathrm{deg}$ Via Ethernet the Macintosh PC was connected to a Windows PC, which was sampling the gaze positions with an EyeLink 1000 eye tracker (Desktop Mount Base System, SR Research, LTD, Canada). The participants were seated $57 \mathrm{~cm}$ in front of the stimulus monitor with their chin fixated supported by a chin rest to minimize head movements.

\section{Eye movement recording}

All experimental sessions were done binocular with only one eye tracked and with a sampling rate of 1000 $\mathrm{Hz}$. Online events as well as all raw gaze position samples were stored on the Windows PC. Online events comprise fixations, saccades and blinks. Additionally we recorded messages corresponding to the course of the program and the mouse clicks. Because all experiments were done in complete darkness it was very important to avoid that the eye illumination from the eye 
tracker was visible. Therefore we have used a system with an illuminator of $910 \mathrm{~nm}$ wavelength, which is out of range of visible light. The system used the pupil and additionally the corneal reflex to detect the corresponding gaze position. Drift checking was performed by showing a fixation spot in the center of the stimulus screen for 5 seconds before and after the adaptation phase. Analyzing this "drift checking" yielded no systematic deviation from our original calibrated fixation spot.

During sampling a saccade event was detected when saccade velocity fell beyond a threshold of $22 \mathrm{deg} / \mathrm{sec}$ which corresponds to an acceleration threshold of 4000 $\mathrm{deg} / \mathrm{sec}^{2}$. Online triggering, which was necessary for the target step, was achieved with a delay between 8 $\mathrm{ms}$ and $16 \mathrm{~ms}$. This delay corresponds to the availability of saccade information on the stimulus computer. All eye movement events were sampled within twice a monitor refresh rate of $16 \mathrm{~ms}$. Because all relevant saccades took at least $40 \mathrm{~ms}$ the target step, necessary for the adaptation procedure, occurred during the eye movement.

\section{Adaptation procedure}

Fixation point (FP) and Target (T1) were red squares with a diameter of $0.6 \times 0.6 \mathrm{deg}$ and a luminance of 0.13 $\mathrm{cd} / \mathrm{m}^{2}$. FP was always $16 \mathrm{deg}$ to the left of the center of the screen. At the start of a trial FP and T1 were displayed on an empty black background and in complete darkness.

After a variable time between 500 and $1300 \mathrm{~ms}$ the fixation point disappeared and subjects had to perform a saccade towards the saccade target $\mathrm{T} 1$, which was in the short saccade experiment 2 deg to the left of the center of the screen, inducing a saccade of $14 \mathrm{deg}$, and in the long saccade experiment $4 \mathrm{deg}$ to the right of the center of the screen, inducing a saccade of $20 \mathrm{deg}$. Subjects were instructed to perform the saccade as fast and as accurately as possible. Before extinguishing FP the tracked eye was required to be within a squared window of $5 \times 5$ deg centered around FP.

While the eyes were moving towards the saccade target T1 and exceeded a threshold of 3 deg rightwards to the fixation point, in the adaptation phase the saccade target T1 stepped outward to T2, which was 6 deg rightwards to T1 (Figure 1). The trigger window at the fixation point ensured that the target was not displaced without a correct saccade from FP towards T1. This allowed that the threshold for target displacement could be placed very close to the fixation point ( $3 \mathrm{deg}$ in our experiment). T2 remained illuminated for $500 \mathrm{~ms}$ after saccade onset. $550 \mathrm{~ms}$ later the next trial began.

The whole adaptation phase consisted of 200 trials. Sixty-six percent of these were proper adaptation trials as described above. We will call these trials pure adaptation trials. The remaining $34 \%$ were trials that did not induce adaptation but that served to prevent

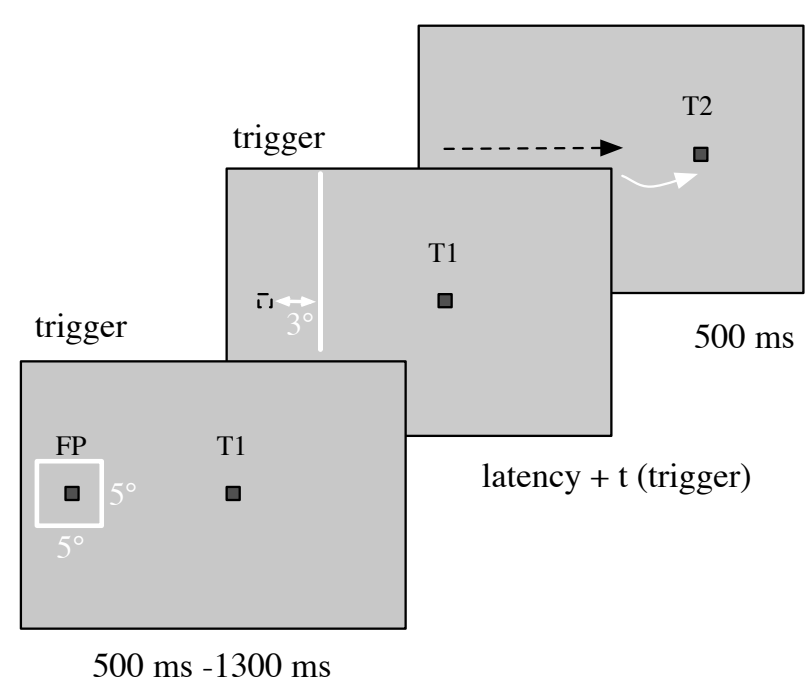

Figure 1. Adaptation procedure. The subject initially fixated FP, which was visible for $500 \mathrm{~ms}$ plus a variable time. Their gaze was triggered to a $5 \times 5$ deg window centered around FP. When FP was extinguished the subject had to perform a saccade to T1. T1 was replaced by T2 as soon as gaze crossed a 3 deg threshold rightwards to FP. T2 was visible for further 500 ms thereafter. In the short saccade experiment the distance from FP to T1 was $14 \mathrm{deg}$. In the long saccade experiment it was $20 \mathrm{deg}$. The distance from T1 to T2 was $6 \mathrm{deg}$ in both experiments.

stereotypic behavior. The target positions were in this case randomly chosen between the vector pair (-12 deg, $10 \mathrm{deg})$ and $(-12 \mathrm{deg},-10 \mathrm{deg})$ thus including a vertical component. In these trials the target was extinguished while the eyes were moving above a $6 \times 6$ deg trigger window centered around FP. We will call all of these trials non-adaptation trials.

\section{Course of experiments}

Each of the subjects participated four times in the short saccade experiment and in the long saccade experiment. To avoid any long-lasting modifications of saccadic eye movements following adaptation (Alahyane \& Pelisson, 2005), we ensured a 7 days break between every experimental session. Before starting with an experimental session the participants were informed in detail about the experimental settings, the course of the experiment, the number of trials and the corresponding approximate duration. Chin rest and chair were adjusted to make experimental conditions as comfortable as possible. The tracked eye was calibrated and validated. In these procedures a successive fixation on 12 dots, that were arranged on a grid, which completed the experimental screen window, and on one dot in the center of the screen, was required.

The experiment started with 34 fixation localization trials. Then 136 mixed target off localization, target on localization, field saccade and standard saccade trials 


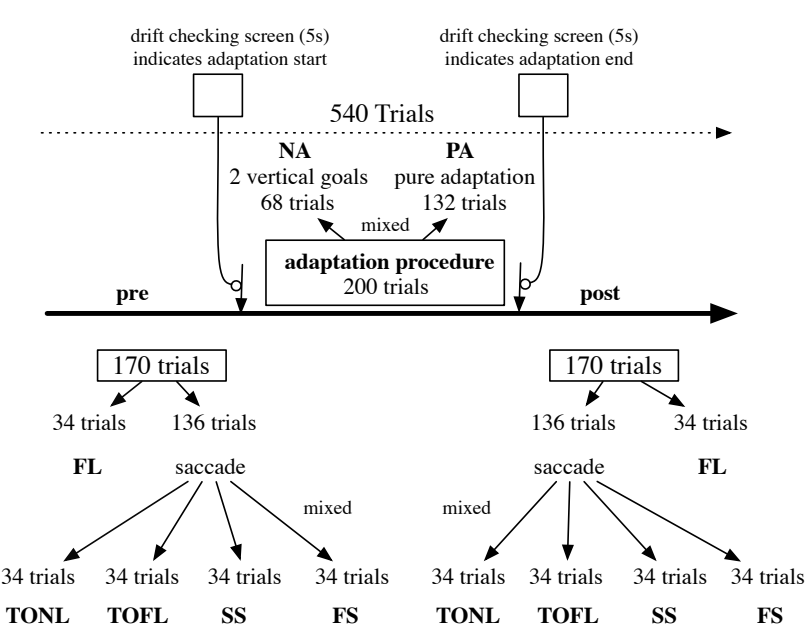

Figure 2. Course of trials in a single experimental session. Abbreviations: $\mathrm{PA}=$ pure adaptation; $\mathrm{NA}=$ non adaptation; $\mathrm{FL}=$ fixation localization; $\mathrm{TONL}=$ target on localization; TOFL = target off localization; $\mathrm{SS}=$ standard saccade; FS = field saccade.

were performed (see below for detailed description). This whole block of 170 trials is the pre-adaptation block, which provides baseline data before adaptation. After the pre-adaptation block the adaptation block started, as described above. The post-adaptation block ended the experiment. It was constructed in the same way as the pre-adaptation block but with the 34 fixation trials at the end of the whole experiment (Figure 2). The 34 testing positions were positioned according to a compact distribution around saccade target and a wide coverage of the 2-dimensional space on the stimulus presentation screen. Many test positions had almost the same horizontal or vertical component as the adapted saccade (see dashed lines in Figure 3). The overall formation of the testing field was a square with 20 deg in horizontal direction and vertical direction (Figure 3). All testing positons were at least $4 \mathrm{deg}$ away from the edges of the screen. Comparison of preand post-adaptation block revealed the influences of outward adaptation to the different test conditions.

\section{Standard saccade trials}

Standard saccade trials in the post-adaptation phase were identical to the pure adaptation trials described in section adaptation procedure. In the post-adaptation phase the 34 standard saccade trials maintained saccadic adaptation. In standard saccade trials in the pre-adaptation phase the target stayed at $\mathrm{T} 1$. Therefore the 34 standard saccade trials occurred in pre- and post-adaptation phase respectively and gave the basic reference for influences of the saccadic adaptation procedure to pure saccade trials.

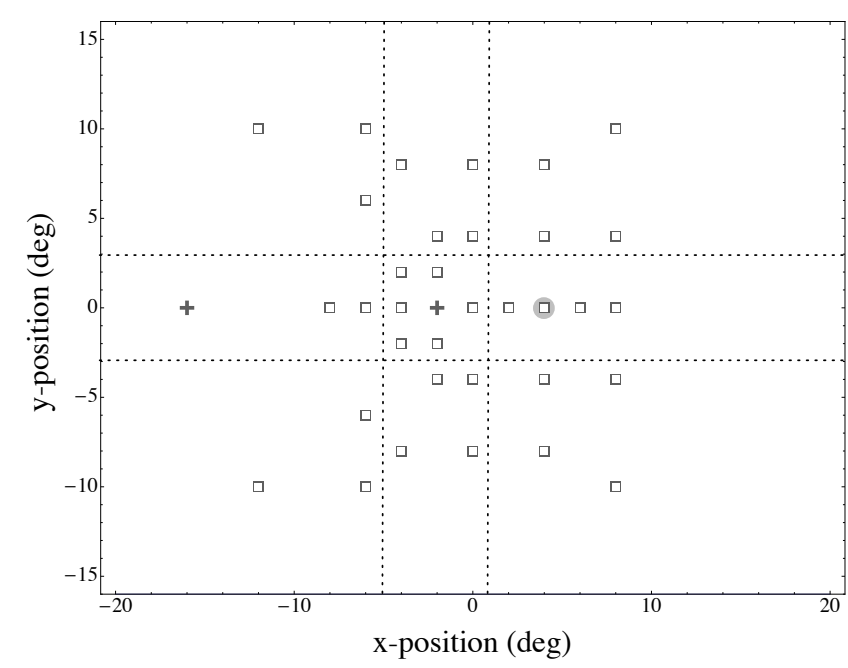

Figure 3. Testing field in the short saccade experiment. Crosses indicate the $14 \mathrm{deg}$ saccade from FP to T1. The filled circle indicates adaptation position T2, 6 deg rightwards from T1. Open squares are the 34 testing positions. For the long saccade experiment all testing positions were $6 \mathrm{deg}$ more to the right.

\section{Field saccade trials}

Thirty-four trials of the pre-adaptation phase and 34 trials of the post-adaptation phase were saccade trials to the 34 testing positions of the adaptation field. We will call these trials field saccade trials. Field saccade trials were identical to the $34 \%$ non adaptation trials in the adaptation phase, except for the different saccade target. In all of these trials the saccadic goal was extinguished during the saccade. The dark screen was visible for further $500 \mathrm{~ms}$ after saccade end. $550 \mathrm{~ms}$ later the next trial began. By measuring saccades to the testing positions before and after saccadic outward adaptation the saccadic outward adaptation field in humans was determined. The difference of saccadic amplitude before and after saccadic adaptation at a test position corresponds to the amount of transfer to that local position.

\section{Target on/Target off localization trials}

The difference between target on localization and target off localization trials was the presence of the saccadic goal as a reference for localization in the target on localization trials.

Target on localization and target off localization trials consisted each of 34 pre- and 34 post-adaptation trials with flashes at the 34 testing positions. In all of these trials subjects had the task to localize a $16 \mathrm{~ms}$ flash with a mouse pointer. The pointer always appeared after a complete trial at a random position and was extinguished after subjects clicked at the perceived position. If subjects were not able to detect any flash they were instructed to click at the left corners of the screen. All 


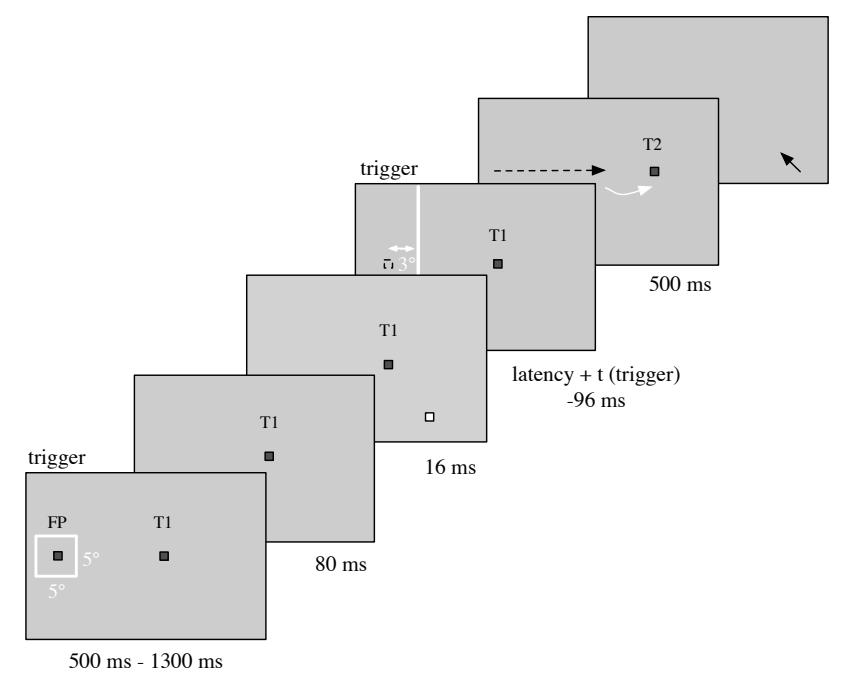

Figure 4. Target on/Target off localization judgements. The subject initially fixated FP, which was visible for a variable time between 500 and $1300 \mathrm{~ms}$. Gaze was triggered to a $5 \mathrm{x}$ 5 deg window centered around FP. As soon as FP was extinguished the subjects had to perform a saccade to T1. Eighty $\mathrm{ms}$ after this go-signal a $16 \mathrm{~ms}$ flash appeared at one of the testing positions. When gaze crossed the threshold 3 deg rightwards to FP T1 jumped to T2 (target on condition) or was extinguished (target off condition, not shown). $500 \mathrm{~ms}$ later a mouse pointer appeared and the subject had to indicate the perceived flash position.

of those clicks were excluded from data analysis afterwards. On the whole those trials were rare $(<1 \%)$. The localization stimulus was a $0.6 \times 0.6 \mathrm{deg}$ square and therefore geometrically identical to our fixation point and target. It was colored in light grey with luminance of $0.10 \mathrm{~cd} / \mathrm{m}^{2}$. Flash presentation in the saccade trials (target on localization/target off localization) occurred always $80 \mathrm{~ms}$ after the fixation point had been extinguished, i.e. after the go-signal for the saccade (Figure 4). Therefore with median latencies of about $240 \mathrm{~ms}$ (see Results) the flash was presented about $160 \mathrm{~ms}$ before saccade onset.

There is a well-known mislocalization for stimuli presented slightly before or during a saccade (Bischof \& Kramer, 1968; Matin, Pearce, \& Pola, 1970; Honda, 1989, 1993; Ross, Morrone, \& Burr, 1997; Kaiser \& Lappe, 2004). However, these peri-saccadic mislocalizations occur only for stimuli flashed within 50 to $100 \mathrm{~ms}$ before the saccade or during the saccade (Honda, 1991; Morrone, Ross, \& Burr, 1997). The adaptation induced mislocalzation that we studied in the present work occurs also for stimuli presented before that time (Awater et al., 2005), and is a perceptual effect separate from peri-saccadic mislocalization (Georg \& Lappe, 2009). To avoid any potential interference of peri-saccadic mislocalization in the present study we presented the localization targets at least $100 \mathrm{~ms}$ before saccade onset.

\section{Fixation localization trials}

Fixation localization trials were performed 34 times in the beginning and in the end of the experiment. The fixation condition was different from all of the other conditions in that no saccade target appeared and no saccade had to be performed. To indicate a fixation trial, the fixation dot was colored violet and a computer voice indicated with the word "fixation" that a fixation trial was starting.

At the beginning of a fixation trial the fixation dot was presented for a variable time between 1500 and 2300 ms. The subject's eye was triggered to a $5 \times 5$ deg trigger window around the fixation dot before the $16 \mathrm{~ms}$ flash appeared at one of the 34 testing positions. After the flash disappeared the fixation dot was visible for further $500 \mathrm{~ms}$ before it was also disappearing. Then, the mouse pointer appeared, and the subject had to localize the corresponding flash position while still fixating (Figure 5). Subjects were instructed to keep their gaze within the primary fixation region until the mouse click. Consequently all judgements had to be done by fixating at a region with $5 \mathrm{deg}$ diameter around the primary fixation dot and therefore from the corner of subject's eye. Within this fixation region only microsaccades less than 0.5 deg were allowed. Trials with saccades larger than $0.5 \mathrm{deg}$ were excluded from analysis.

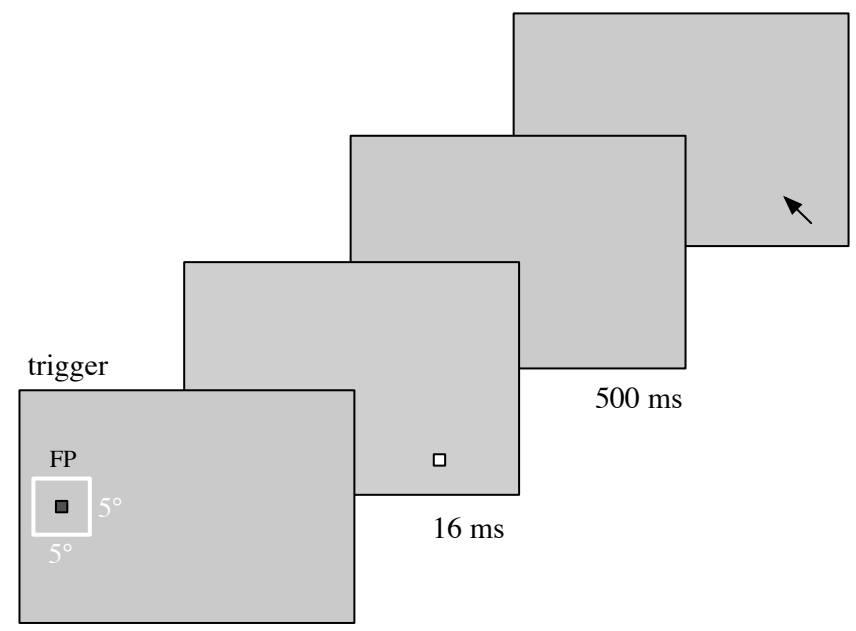

$1500 \mathrm{~ms}-2300 \mathrm{~ms}$

Figure 5. Fixation localization judgements. The subject fixated FP for a variable time between 1500 and 2300 ms. Gaze was triggered to a $5 \times 5$ deg window centered around FP. A computer voice and the slightly violet color of FP instructed the subject that a fixation trial was starting. Then a $16 \mathrm{~ms}$ long flash appeared at one of the 34 testing positions. Subjects had to maintain fixation until after the localization report with the mouse pointer was completed. 


\section{Data Analysis}

Mathematica 7.0 was used for all data analysis. Saccade amplitude analysis was based on the saccade parameters estimated by the Eyelink software. Only the first saccade after the go-signal was used for data analysis. For a saccade to enter analysis, its start point had to be within a circle of $5 \mathrm{deg}$ diameter around the fixation point, its amplitude had to be between $5 \mathrm{deg}$ and $40 \mathrm{deg}$ and its duration had to be between $20 \mathrm{~ms}$ and $100 \mathrm{~ms}$ in the short saccade experiment and between 30 and $110 \mathrm{~ms}$ in the long saccade experiment. Based on these criteria $95.7 \% \pm 2.3$ (SD) in the short saccade experiment and $92.5 \% \pm 7.8(\mathrm{SD})$ in the long saccade experiment of all saccades in the adaptation phase and in the standard saccade, target on localization, and target off localization conditions were accepted for data analysis. There was no significant difference in saccade acceptability between the target on localization, target off localization and standard saccade conditions (short saccade experiment: $F_{2,15}=0.007, p=0.993$; long saccade experiment: $\mathrm{F}_{2,15}=0.03, \mathrm{p}=0.970$ ). The above mentioned criteria also hold for the field saccade trials, except for higher upper thresholds in the amplitude dependent criterion (50 deg in short saccade experiment; 60 deg in long saccade experiment) and higher upper thresholds in saccadic duration (120 ms in short saccade experiment; $150 \mathrm{~ms}$ in long saccade experiment). For these field saccade trials an accuracy criterion was additionally implemented. Saccades that missed the target by more than $10 \mathrm{deg}$ were excluded from data analysis (error rate $<1 \%$ ). With this criterion and the above mentioned saccade criteria $95.0 \% \pm 2.9$ (SD) of all the field saccade trials in the short saccade experiment and $91.6 \% \pm 6.9$ (SD) of all the field saccade trials in the long saccade experiment were accepted.

Analysis of the saccadic transfer within the saccadic adaptation field was done by taking the median over all four experimental sessions of one subject at one position and then taking the mean over all six subjects for that position. This procedure was done separately for pre-adaptation and post-adaptation trials.

For the analysis of the fixation localization trials first all saccades in a fixed time window starting $1500 \mathrm{~ms}$ after FP onset and ending with the active mouse click were determined. The first fixation localization criterion was an adequate fixation within a 5 deg diameter circle around FP. All detectable saccadic events had to be shorter than $0.5 \mathrm{deg}$, implicating that only small saccades or microsaccades were allowed within the fixation region. In four of six subjects microsaccades longer than 0.5 deg occured in less than $20 \%$ of all fixation trials. In one subject microsaccades longer than $0.5 \mathrm{deg}$ occured in less then $40 \%$ of the fixation trials. However in one subject we had to discard about $60 \%$ of all fixation trials because of major microsaccadic events. The values hold for the short saccade as well as the long saccade experiment. A second fixation localization analy- sis criterion was related to the localization judgements of the flashes. Fixation localization trials were only accepted when subjects localized the flash within a circle of $10 \mathrm{deg}$ diameter around its true position. With the above criteria $73.5 \% \pm 20.0$ (SD) of all fixation localization trials in the short saccade experiment and $73.2 \%$ $\pm 26.1(\mathrm{SD})$ of all fixation localization trials in the long saccade experiment were accepted. For data analysis the median of the four experiments of each subject's localization judgements was computed at each flash location and the resulting data was averaged over all subjects. The procedure was done separately for pre- and post-adaptation trials.

In the target on localization and target off localization trials the saccade criteria mentioned at the beginning of this section were combined with the just mentioned localization judgement criterion. Following these criteria $92.0 \% \pm 4.1$ (SD) of all the target on localization trials in the short saccade experiment and 92.2\% \pm 6.7 (SD) of all the target on localization trials in the long saccade experiment were accepted while in the target off localization condition $92.3 \% \pm 4.0$ (SD) of all the trials in the short saccade experiment and $91.5 \% \pm 6.9$ (SD) of all the trials in the long saccade experiment were accepted.

\section{Results}

We investigated the adaptation field and the corresponding mislocalization field in saccadic outward adaptation in humans for a $14 \mathrm{deg}$ saccade (short saccade experiment) and a $20 \mathrm{deg}$ saccade (long saccade experiment). We will first describe the adaptation field results for both experiments and then the corresponding mislocalization fields and their correlation to the adaptation field.

\section{Adaptation in the short saccade experiment}

An example of the adaptation in the short saccade experiment is shown in Figure 6a. Averaged over subjects and trial types the mean increase of saccade amplitude, i.e. the adaptation gain, was $13.2 \% \pm 1.2$ (SE). The adaptation gain was defined as

$$
\text { gain }_{\%}=\frac{\text { amplitude }_{\text {post }}-\text { amplitude }_{\text {pre }}}{\text { amplitude }_{\text {pre }}} \cdot 100 .
$$

The average saccade latency was $240.9 \mathrm{~ms} \pm 18.5$ (SD). A one-dimensional ANOVA revealed no significant differences in latencies between pre-adaptation, adaptation and post-adaptation trials $\left(\mathrm{F}_{2,15}=2.77, \mathrm{p}=0.095\right)$. Latencies between standard saccade, target on localization, and target off localization trials were also not different from each other $\left(\mathrm{F}_{2,15}=2.27, \mathrm{p}=0.137\right)$.

We fitted an exponential function $\mathrm{f}(\mathrm{n})=\mathrm{a}-\mathrm{b} / \exp (\mathrm{c} \cdot \mathrm{n})$ to the adaptation curve (all horizontal trials in the adaptation phase) to investigate the rate constant $c$ 

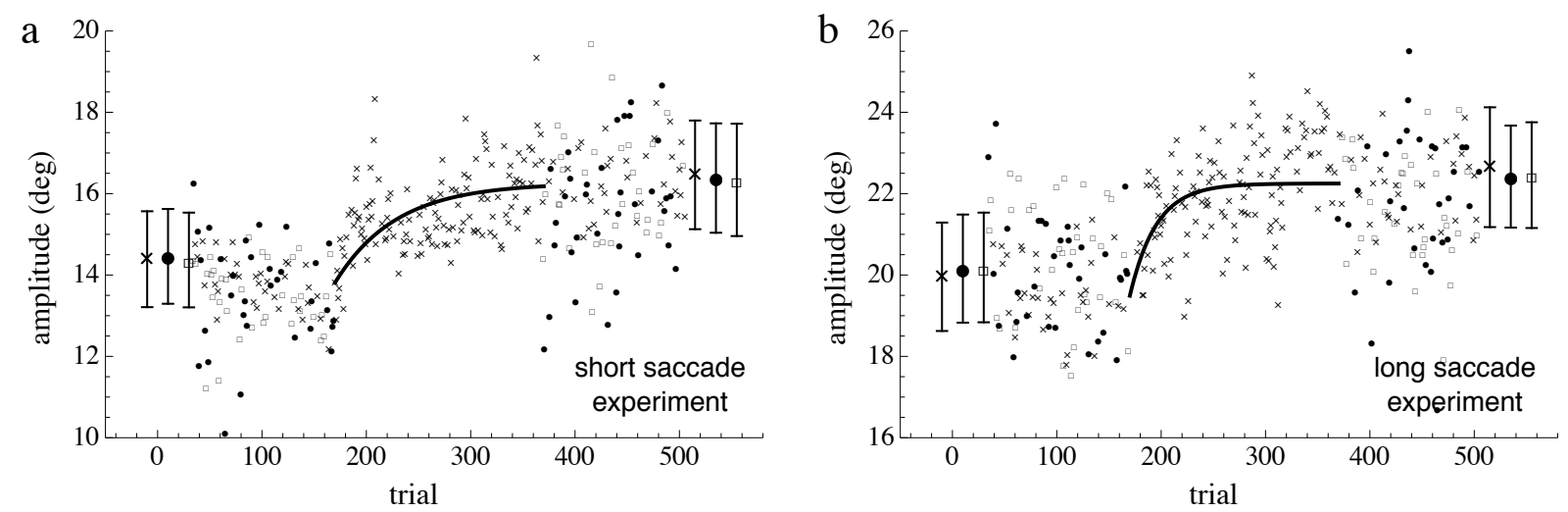

Figure 6. Examples of adaptation over an individual session in the short saccade (a) and long saccade (b) experiment. The figures show saccade amplitude as a function of trial number. Crosses indicate the pure saccade trials (pure adaptation and standard saccade), filled circles indicate the target on localization trials and open squares indicate the target off localization trials. The large symbols on the edges of each figure indicate the mean saccade amplitude over all subjects in the pre-adaptation (left edge) and post-adaptation (right edge) phases. Error bars are standard deviations (SD).

which measures the speed of adaptation (Miller et al., 1981; Fujita, Amagai, Minakawa, \& Aoki, 2002). In this exponential function a describes the final amplitude, $b$ the amount of adaptation, and the difference $a-b$ consequently the initial amplitude. The initial amplitude of each subject was computed by taking the median of all data points in the pre-adaptation phase. The mean initial amplitude over all subjects was $14.3 \mathrm{deg} \pm 0.4$ (SE). The final amplitude of each subject was computed by taking the median of all data points in the postadaptation phase. The mean over all subjects was 16.3 $\operatorname{deg} \pm 0.4$ (SE). Consequently the amount of adaptation $b$ was 2 deg $\pm 0.1(\mathrm{SE})$ and therefore one third of the original 6 deg target step.

All data were then fitted with the individual parameters of each experimental session. This gave a mean rate constant over all subjects of $c=0.10 \pm 0.05$ (SE). The rate constant is in good accordance with the value of 0.11 that was obtained for outward adaptation in the study of Miller et al. (1981). Figure 6a shows the fitted exponential time course for the adaptation of that particular subject.

Before saccadic adaptation the mean duration of the 14 deg saccade was $53.4 \mathrm{~ms} \pm 5.6$ (SD). After outward adaptation the duration increased to $57.4 \mathrm{~ms} \pm 6.6$ (SD) $(\mathrm{p}<0.01)$. A one-dimensional ANOVA revealed no significant differences in duration increase between standard saccade, target on localization and target off localization trials $\left(\mathrm{F}_{2,15}=0.23, \mathrm{p}=0.799\right)$. Mean saccadic peak velocity was $490 \mathrm{deg} / \mathrm{s} \pm 30$ (SD) in the preadaptation phase and $510 \mathrm{deg} / \mathrm{s} \pm 40$ (SD) in the postadaptation phase.

\section{Adaptation in the long saccade experiment}

Figure $6 \mathrm{~b}$ shows an example of the adaptation in the long saccade experiment. The mean adaptation gain over all subjects was $13.5 \% \pm 1.0$ (SE). The average saccade latency was $234.8 \mathrm{~ms} \pm 15.5$ (SD). No latency differences were found between pre-adaptation, adaptation and post-adaptation phase $\left(\mathrm{F}_{2,15}=1.67, \mathrm{p}=0.221\right)$ as well as between standard saccade, target on localization, and target off localization conditions $\left(\mathrm{F}_{2,15}=0.84\right.$, $\mathrm{p}=0.452)$. The fitting procedure was the same as in the short saccade experiment. The mean initial amplitude was $20.0 \mathrm{deg} \pm 0.6$ (SE). The mean final amplitude was $22.7 \mathrm{deg} \pm 0.6(\mathrm{SE})$. Consequently the amount of adaptation $b$ was $2.7 \mathrm{deg} \pm 0.2$ (SE) and therefore about half of the original 6 deg target step. A mean rate constant of $c=0.15 \pm 0.08$ (SE) resulted from the exponential fitting. Comparison between the rate constant $c$ in the short saccade and long saccade experiment did not reveal a significant difference. Nevertheless, the longer $20 \mathrm{deg}$ saccade was more adapted $(45 \%$ of the original target step) compared with the shorter 14 deg saccade (33\% of the original target step).

Before saccadic adaptation, mean duration was 64.7 $\mathrm{ms} \pm 8.4$ (SD). After outward adaptation duration increased to $71.1 \mathrm{~ms} \pm 8.4$ (SD) corresponding to a significant duration increase of $6.4 \mathrm{~ms} \pm 1.2$ (SE) ( $\mathrm{p}<$ 0.005). A one-dimensional ANOVA revealed no significant differences in duration increase between standard saccade, target on localization, and target off localization trials $\left(\mathrm{F}_{2,15}=0.88, \mathrm{p}=0.434\right)$. Mean saccadic peak velocity was in the pre-adaptation phase $540 \mathrm{deg} / \mathrm{s} \pm$ $0.02(\mathrm{SD})$ and in the post-adaptation phase also 540 $\mathrm{deg} / \mathrm{s} \pm 0.02$ (SD).

\section{Saccadic adaptation fields}

To measure the transfer of adaptation to the 34 testing positions we computed the median of the four experimental sessions of each subject in the pre- and in the post-adaptation phase at each local position of the 

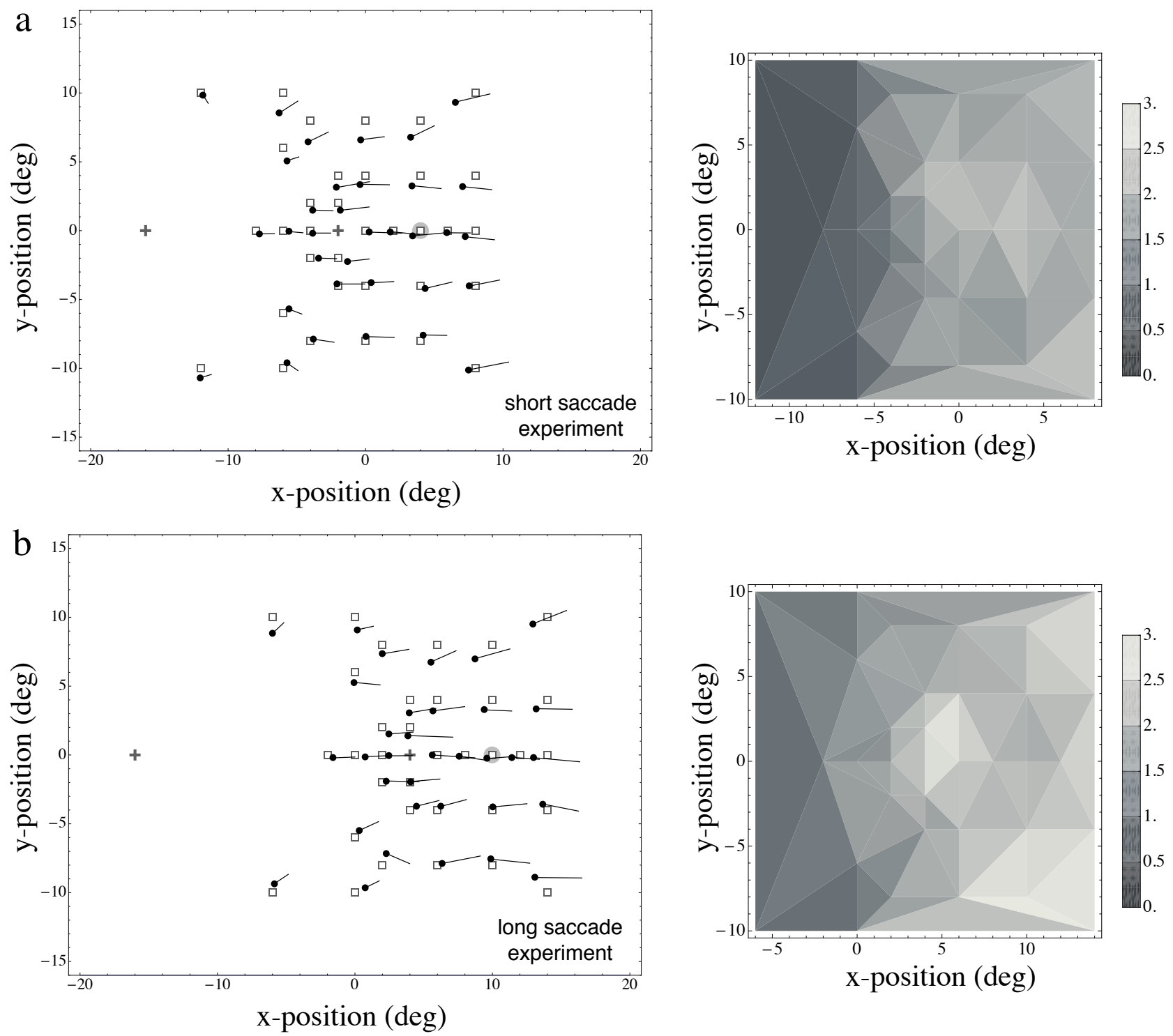

Figure 7. a: Saccadic adaptation field for the $14 \mathrm{deg}$ adapted saccade. b: Saccadic adaptation field for the 20 deg adapted saccade. Crosses indicate FP and T1 while the bright circle indicates T2. Open Squares are testing positions. Black disks represent saccade endpoints in the pre-adaptation phase. The lines point towards the saccade endpoints of the corresponding eye movements in the post-adaptation phase. Brightness in the heat maps represents amount of adaptation at a particular testing position.

adaptation field, and then took the mean over all subjects. The adaptive shift was calculated as the difference between the mean in the post-adaptation phase and the mean in the pre-adaptation phase at a particular field position. Figure 7 shows the adaptation fields in the short saccade and long saccade experiment. The mean adaptive shift over all 34 local positions was $(\mathrm{x}=$ $1.70 \mathrm{deg} \pm 0.26$ (SD), $\mathrm{y}=0.07 \mathrm{deg} \pm 0.17$ (SD)) in the short saccade and $(x=2.06 \mathrm{deg} \pm 0.26(\mathrm{SD}), \mathrm{y}=0.08$ $\mathrm{deg} \pm 0.22(\mathrm{SD})$ ) in the long saccade experiment and in both cases significantly different from zero (short sac- cade experiment: paired t-test, $\mathrm{p}<0.0005$; long saccade experiment: paired t-test, $\mathrm{p}<0.0005)$. Besides the mean adaptive shift in the short saccade and long saccade experiment were significantly different from each other (paired t-test, $\mathrm{p}<0.05$ ).

Looking upon the adaptation fields qualitatively, the distribution of the adaptive shift over the 34 tested locations appears asymmetric in the horizontal direction, as it increases with increasing distance from the fixation point. In the vertical direction, the adaptive shift appears symmetric and with only a shallow peak 

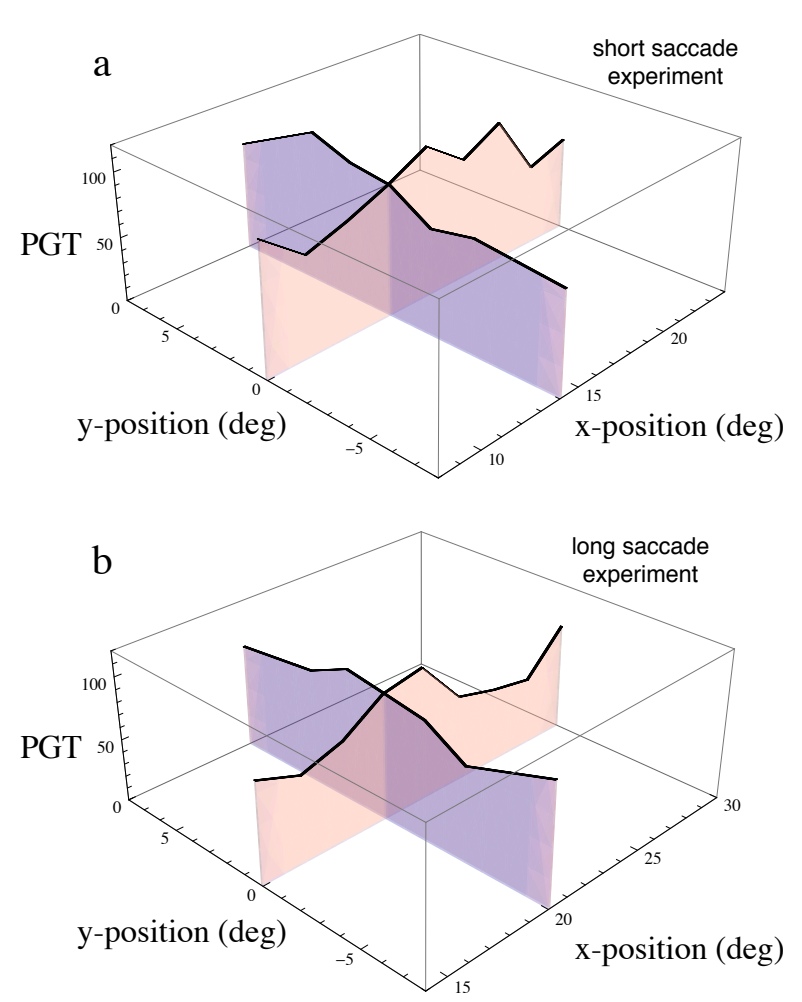

Figure 8. Percent gain transfer for saccades to testing positions which share almost the same vertical and horizontal components as the adapted 14 deg saccade (a) or the adapted 20 deg saccade (b). The brighter surface indicates the horizontal component and the darker surface the vertical component.

around the adapted saccade, as only slight deviations are observable. Therefore the global appearance of the adaptive shift distribution in the range of our testing positions is that of a uniform planar increase rather than a sharp peak at the adapted amplitude. However, the two points above and below the horizontal meridian close to the fixation point show much less adaptation, consistent with earlier findings that the adaptation transfer is limited to around $\pm 30 \mathrm{deg}$ around the adapted direction (Deubel, 1987).

To get a deeper impression of the amount of adaptive eye position shift Figure 7 shows a so called heat map of the adaptive shifts in the saccadic adaptation field. The two dimensional map represents horizontal and vertical components of the adaptation field while the amount of transfer is represented through a grayscaled projection onto the two dimensional map. Because of a finite number of testing positions in the adaptation field (34 local positions) a 2 dimensional map is constructed throughout interpolations between the testing positions.

Another way to look at the saccadic adaptation field is in terms of percent gain transfer (PGT) of the adapted saccade $\mathrm{j}$ to each testing position $\mathrm{i}$ according to formula

$$
\operatorname{PGT}_{\mathrm{ij}}=\frac{\operatorname{gain}_{\%}(\mathrm{i})}{\operatorname{gain}_{\%}(\mathrm{j})} \cdot 100 .
$$

The percent gain transfer $\mathrm{PGT}_{\mathrm{ij}}$ describes how much a saccade to position $\mathrm{i}$ increases in gain in proportion to the gain increase at the adapted site $\mathrm{j}$. Unlike the adaptation shift, which measures absolute changes in saccade amplitude, the percent gain transfer measures changes relative to the gain change of a particular saccade amplitude (typically the saccade amplitude of the adapted saccade). Figure 8 shows the percent gain transfer for saccades sharing almost the same vertical or horizontal components as the adapted saccade in a 3-dimensional plot. The figure shows that the percent gain transfer depended on the distance of the testing positions from the adapted $14 \mathrm{deg}$ (short saccade experiment) or $20 \mathrm{deg}$ (long saccade experiment) saccade. Gain was slightly higher for the adapted saccade than for saccades with increasing distance from the adapted saccade. However, overall the amount of gain transfer in saccadic outward adaptation was rather high at all positions tested.

\section{Localization judgements}

Figures 9a and b show localization judgements in the target on condition. The mean outward mislocalization shift over all 34 local positions was $(x=2.46 \mathrm{deg} \pm 0.60$ (SD), $y=0.01 \mathrm{deg} \pm 0.21(\mathrm{SD}))$ in the short saccade and $(x=2.53 \mathrm{deg} \pm 0.91(\mathrm{SD}), \mathrm{y}=0.05 \mathrm{deg} \pm 0.16(\mathrm{SD}))$ in the long saccade experiment. The horizontal shift was in both experiments significantly different from 0 (short saccade experiment: paired t-test, $\mathrm{p}<0.0005$; long saccade experiment: paired t-test, $\mathrm{p}<0.005)$.

Looking upon the resulting mislocalization fields qualitatively the target on mislocalization fields appear asymmetric along the horizontal axis with increasing mislocalization for targets further away from the fixation point. Along the vertical axis the mislocalizations appear symmetric with larger mislocalization near the horizontal axis and smaller mislocalization at positions farther away from this axis. Thus, the mislocalization field, like the adaptation field, appears spatially nonuniform. A comparison of the heat maps of mislocalizations in the target on condition and the adaptation field in both experiments emphasizes the similarities.

Figures $9 \mathrm{c}$ and $\mathrm{d}$ show the corresponding mislocalization fields in the target off condition. In the absence of visual references (target off) the mean over all 34 localization judgements in the post-adaptation phase shifted significantly outwards by $(x=1.23 \mathrm{deg} \pm 0.59$ (SD), $y=0.01 \mathrm{deg} \pm 0.31$ (SD), paired t-test, $\mathrm{p}<0.005)$ in the short saccade experiment and $(x=1.51 \mathrm{deg} \pm 0.67$ (SD), $y=-0.01 \mathrm{deg} \pm 0.26(\mathrm{SD})$, paired t-test, $\mathrm{p}<0.005)$ in the long saccade experiment. Thus, the outward mislocalization is smaller in the target off condition than in 

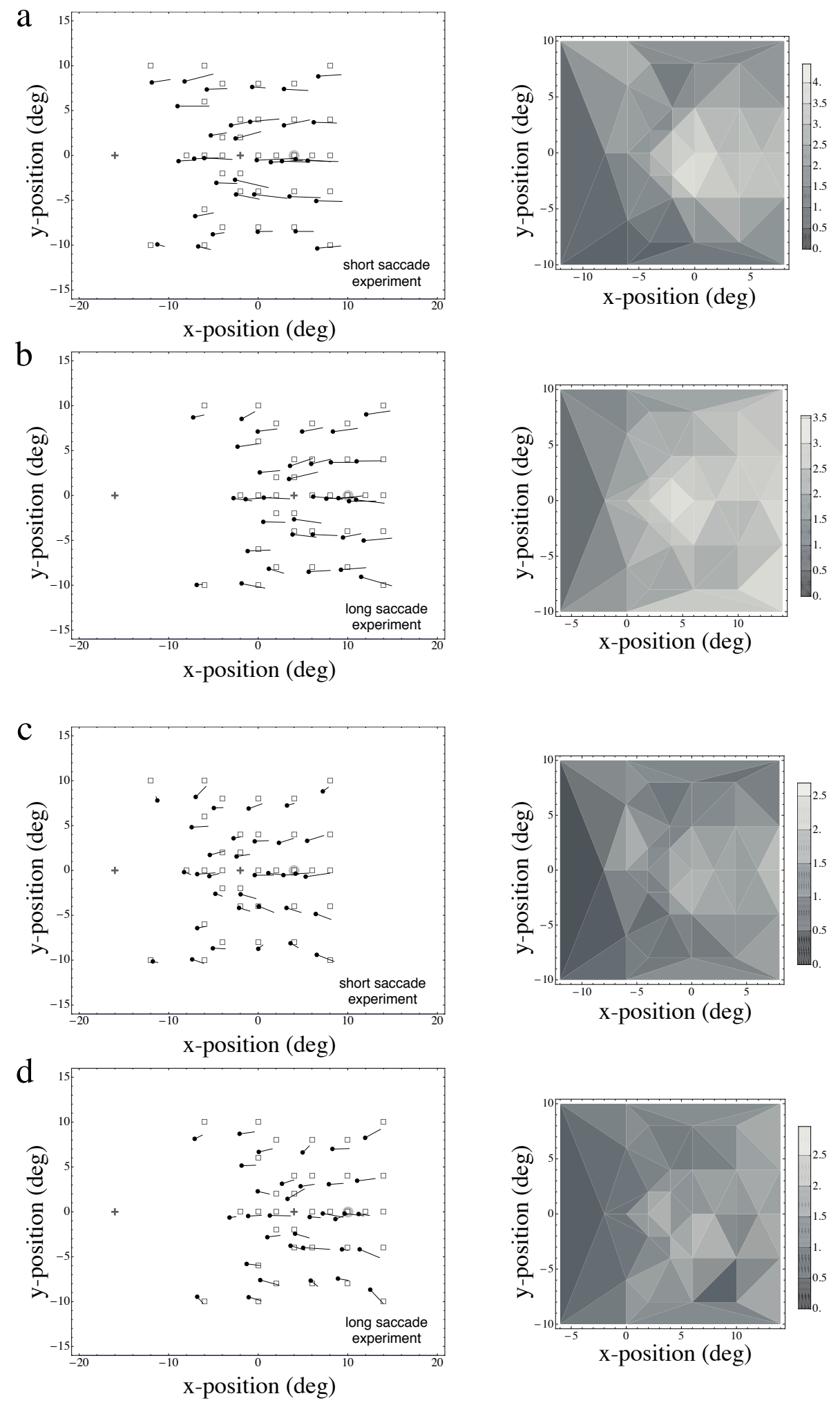

Figure 9. Localization judgements. Target on localization condition in the short saccade experiment (a) and long saccade experiment (b). Target off localization condition in the short saccade experiment (c) and long saccade experiment (d). Filled circles indicate localization judgements in the pre-adaptation phase while the line ends indicate the corresponding judgements in the post-adaptation phase. All figures are with their corresponding heat maps. 
the target on condition. Moreover, the mislocalization fields in the target off localization condition seem to be more radial than in the target on localization condition. Together with the smaller overall mislocalization this could suggest that mislocalization in the target on condition resulted from mislocalization in the target off condition plus an additional parallel shift, based upon the post-saccadic view of the target.

We quantitatively compared the amount of mislocalization with the amount of saccadic adaptation by computing the correlation between mislocalization at each position in the testing field and the corresponding saccade amplitude to that position. Figure 10a and $\mathrm{b}$ reveal in both experiments a positive correlation between the mislocalization $\mathrm{M}$ at a given position and the corresponding amount of adaptive shift $\mathrm{S}$ at this position for the target on condition (short saccade experiment: $\mathrm{M}=$ $1.69+0.54 \cdot \mathrm{S}, \mathrm{R}^{2}=0.14, \mathrm{p}<0.05$; long saccade experiment: $\left.\mathrm{M}=1.24+0.60 \cdot \mathrm{S}, \mathrm{R}^{2}=0.41, \mathrm{p}<0.01\right)$. A significant correlation is also observed in the target off condition for the short saccade experiment, but in the long saccade experiment no significant correlation is observable (short saccade experiment: $\mathrm{M}=0.84+0.34 \cdot \mathrm{S}, \mathrm{R}^{2}$ $=0.13, \mathrm{p}<0.05$; long saccade experiment: $\mathrm{M}=1.35+$ $0.12 \cdot S, R^{2}=0.02, p=0.41$ ). Thus the localization of the flash somewhat depends on the metrics of the saccade, in accordance to the findings of Collins et al. (2007) for saccadic inward adaptation. However our correlations are smaller than those for saccadic inward adaptation, suggesting that motor space and perceptual space are linked to each other but that they are not identical.

The above analysis shows a correlation between the mislocalization at a particular position and the adaptation of saccades made to that particular. However, in the trials in which the mislocalization was measured the saccade was not directed to the tested localization position but was a standard saccade to the adapted target, i.e. 14 deg to the right in the short saccade experiment and 20 deg to the right in the long saccade experiment. Thus, one might ask whether the amount of mislocalization also correlates with the amount of adaptation of the saccade that was actually performed in the localization trials. The correlation analysis gave no significant correlation between the mislocalization $\mathrm{M}$ during a localization trial and the amount of the adaptation shift $S_{P}$ of the performed saccade in any of the experiments (long saccade experiment: target on localization: $\mathrm{M}=2.45+0.04 \cdot \mathrm{S}_{P}, \mathrm{R}^{2}<0.005, \mathrm{p}=0.84$; target off localization: $\mathrm{M}=1.46+0.07 \cdot \mathrm{S}_{P}, \mathrm{R}^{2}<0.005, \mathrm{p}=0.71$; short saccade experiment: target on localization: $\mathrm{M}=2.18+$ $0.24 \cdot \mathrm{S}_{P}, \mathrm{R}^{2}<0.05, \mathrm{p}=0.42$; target off localization: $\mathrm{M}=$ $\left.0.77+0.31 \cdot \mathrm{S}_{P}, \mathrm{R}^{2}<0.1, \mathrm{p}=0.09\right)$. Thus, like in the case of inward adaptation (Collins et al., 2007) mislocalization at a particular target position is not coupled to the adaptation of the saccade that is actually performed.
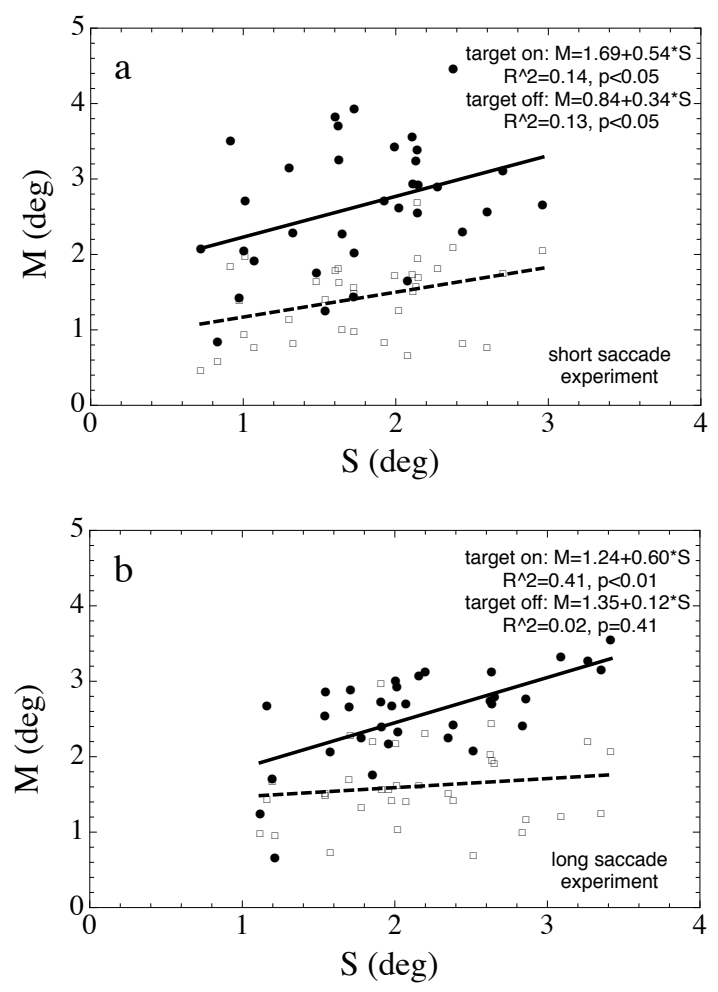

Figure 10. Correlations. Correlation between mislocalization $\mathrm{M}$ at each position in the testing field and the corresponding amount of adaptive shift $S$ at that position in the short saccade experiment (a) and in the long saccade experiment (b). Filled circles indicate target on localization trials and open squares target off localization trials.

\section{Localization during fixation}

Every experimental session started and ended with 34 fixation localization trials. In these trials subjects had to fixate throughout the trial. Subjects were not allowed to do any saccade during these trials, i.e., they had to localize the flashes from the corner of their eyes. Figure 11 shows the localization differences between before and after adaptation, calculated by taking the median of each subject's localization judgements at one of the testing positions in pre- and post-adaptation phase respectively and then subtracting the mean over all subjects in pre-adaptation phase from the mean in post-adaptation phase. The mislocalizations during fixation were much smaller than when a saccade was performed. However, the mean over all 34 positions and the mean over all subjects gave a significant outward shift of $0.71 \pm 0.50$ (SD) (one-sample t-test, $p$ $<0.05)$ deg in the short saccade experiment. In the long saccade experiment no significant shift was observed $(-0.11 \pm 0.89 \mathrm{deg}$; one-sample t-test, $\mathrm{p}>0.05)$. 

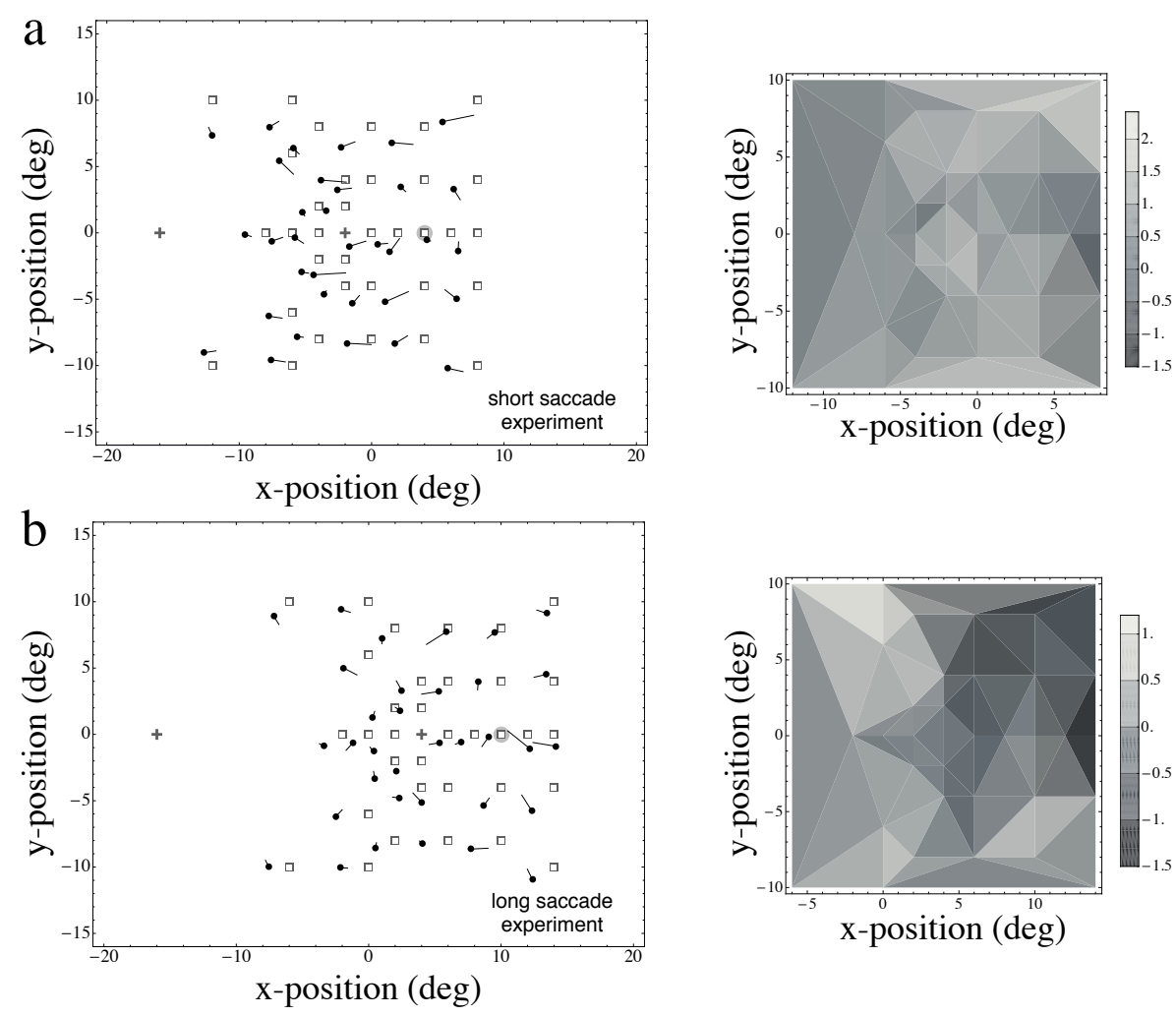

Figure 11. a: Fixation localization judgements in the short saccade experiment. b: Fixation localization judgements in the long saccade experiment. Both figures are with their corresponding heat maps. Filled circles indicate the mean of fixation localization judgements in the pre-adaptation phase and end of the line the mean of fixation localization judgements in the post-adaptation phase.

\section{Discussion}

\section{The human outward adaptation field}

We measured the two-dimensional adaptation field in humans for saccadic outward adaptation. We found rather strong transfer of the adaptation of one saccade to the amplitudes of saccades to neighboring spatial locations. The absolute change of saccade amplitude declined for saccades to targets closer to the fixation point than the adapted saccades and increased for saccades to targets further away than the adapted target. Target location along the vertical dimension in the range of our testing positions had less influence on the amount of adaptation than in saccadic inward adaptation (Collins et al., 2007). For a particular horizontal amplitude saccades to targets at different vertical locations all showed rather similar amplitude change. Thus, the outward adaptation field in humans seems rather planar directly around the adapted saccade with an almost linear increase along the horizontal axis and high symmetry along the vertical axis.

This planar appearance of the adaptation field in this part of oculomotor space is not inconsistent with a limited transfer of adaptation for saccade direction (Deubel, 1987). Our test positions were arranged to fill a 20 deg times 20 deg square around the adapted saccade. Limits of adaptation transfer should be expected at \pm 30 deg around the adapted saccade direction (Deubel, 1987), i.e. outside our testing area. Some indication of the decline of transfer can be seen at the edges of our testing space, particularly for the locations above and below the meridian that are closest to the fixation point. Also, our data are compatible with a vectorial transfer of adaptation. Qualitatively one can indeed observe a radial component of the shifts in the outward adaptation fields. The adaptation fields in our study may result from a vector addition of a radial and a parallel shift vector. Furthermore, the linear transfer of the effect of adaptation with increased saccade size is compatible with the assumption of a parametric gain model with a single gain parameter for a large range of amplitudes (Deubel, 1991).

We also calculated the saccadic gain transfer as a percentage of target amplitude. This calculation revealed a broader gain transfer field than it was found in saccadic inward adaptation (Collins et al., 2007) with a slight maximum at the position of the adapted saccade 
and with little decline in any direction. The percentage change of saccade amplitude declined slowly and symmetrically in all direction from the adapted target location. This is different from studies in monkeys that showed a rather narrow percent gain transfer field for saccadic outward adaptation with maximum transfer near the position of the adapted saccade (Noto et al., 1999; Watanabe, Noto, \& Fuchs, 2000). The gain changes in our study show a relative high amount of transfer of the outward adapted saccade to the other positions in the testing field. There is still about $80 \%$ gain transfer if the saccade goal is more than $8 \mathrm{deg}$ away from the adapted saccade (Figure 8). This again emphasizes the planar appearance and the almost linear increase in the horizontal direction of the corresponding outward adaptation field. Moreover the gain transfer field is symmetric along the vertical and the horizontal direction. A symmetric gain transfer was also found in one out of two monkeys in the study of Noto et al. (1999), but the other monkey had an asymmetrical gain transfer field along the horizontal direction. The difference in the amount of gain transfer in the outward adaptation field between monkeys and humans could highlight a species difference in adaptation processes.

Two earlier studies have investigated spatial transfer to a small number of other saccade amplitudes for human outward adaptation. Those studies investigated percent gain transfer to testing positions on the horizontal axis and found a rather narrow gain transfer for saccadic outward adaptation (Miller et al., 1981; Semmlow et al., 1989). Miller et al. (1981) tested adaptation transfer to a saccade shorter than the adapted one and to a saccade longer than the adapted one after saccadic outward adaptation. The test positions were 4 deg after adaptation of a 8 deg saccade with a 4 deg outward step and 6 deg after adaptation of a 4 deg saccade with a 2 deg outward step. Average gain transfer was about $43 \%$ across both testing saccades, suggesting a steep decline of adaptation away from the adapated amplitude. However, they noted that statistical significance of their results was marginal because of the small number of subjects and the small amount of adaptation. Semmlow et al. (1989) reported results of outward adaptation of a $8 \mathrm{deg}$ saccade with a 8 deg target step, which resulted in about 2 deg of adaptation. They found nearly no transfer to a $16 \mathrm{deg}$ saccade and about $50 \%$ transfer to saccades of $4 \mathrm{deg}$ or $12 \mathrm{deg}$. Our results in the short saccade and long saccade experiment raise speculations about a broader gain transfer field in two dimensions with a flat gain transfer maximum at the position of the adapted saccade and strong gain transfer at positions even far away from the adapated saccade target. One difference between our study and the studies of Miller et al. (1981) and Semmlow et al. (1989) is the adapted saccade length. It was comparatively short in the former studies (Miller: 4 deg and 8 deg; Semmlow: $8 \mathrm{deg}$ ) and much larger in our short saccade (14 deg) and long saccade (20 deg) experiment. Influences of the adapted saccade size on the adaptation field geometry and shape were already observed by Noto et al. (1999) for saccadic inward adaptation in the monkey. Although gain transfer in their study was smaller at all, which indeed could be due to species differences or differences between saccadic inward and outward adaptation, they revealed a sharper peak in the vicinity of the adapted saccade for shorter adapted saccade lengths than for the longer ones (Noto et al., 1999). A second difference is that we included many more testing positions than the previous studies. Therefore the number of different saccades with different saccade length is more than eight times higher than in the experiment by Semmlow et al. (1989) and more than fifteen times higher than in the Miller et al. (1981) study. Many different testing positions in our study could effect the transfer rate by counteracting particular forms of fatigue, that ocurr in stereotypic perfomance of identical saccades for a long time (Chen-Harris et al., 2008). The need to make many different saccades in our experiment would make fatigue occurance less likely. Fatigue effects would be particularly troublesome in saccadic outward adaptation because they act contrary to the original saccade step and therefore can even influence the transfer rate. Third, our study used an overlap paradigm in which the saccade target is present for some time while the subject is still fixating such that subjects have got full information about the location of the saccade target before the go signal for the saccade is given by extinguishing the fixation point. This type of experiment is often linked to voluntary saccade generation (Pierrot-Deseilligny, 1991; Hopp \& Fuchs, 2004). The studies by Miller et al. (1981) and Semmlow et al. (1989) used a reactive saccade paradigm in which subjects have to perform the saccade immediately when the target is turned on. Reactive and voluntary saccades differ in their transfer rates for inward adaptation (Deubel, 1995; Collins \& Dore-Mazars, 2006; Cotti, Guillaume, Alahyane, Pelisson, \& Vercher, 2007) and in the induction of mislocalization (Zimmermann \& Lappe, 2009).

Our analysis shows both similarities and differences between the human outward adaptation field (Figure 7) and the human inward adaptation field measured by Collins et al. (2007). Like inward adaptation, outward adaptation transfers to neighboring target locations in both horizontal and vertical directions (Figure 7 ). In both cases the adaptation field is symmetric in the vertical direction and asymmetric in the horizontal direction. However, inward and outward adaptation fields could differ in their geometry. The outward adaptation field seems to be rather planar with a continuous increase along the horizontal direction and not much difference along the vertical direction at least in the range of our testing positions. The inward adaptation field is more peaked with strongest adaptation at the adapted saccade amplitude and de- 
creasing adaptation at neighboring amplitudes (Collins et al., 2007). This is highlighted by the gain transfer field (Figure 8) for saccades sharing almost the same vertical or horizontal component as the adapted saccade. Gain transfer rates are large and symmetric in all transfer directions for saccadic outward adaptation whereas they drop quickly symmetric in vertical transfer direction and asymmetric in horizontal one for saccadic inward adaptation (Collins et al., 2007). Comparison of the inward and outward gain transfer fields between humans and monkeys (Noto et al., 1999) could reveal that the inward gain transfer fields of both primates are very similar (narrow, asymmetric in horizontal and symmetric in vertical direction), but the outward gain transfer field is broader and more symmetric in humans than in monkeys. Comparison of the adaptation fields for 14 and 20 deg saccades showed that the adapted saccade length did not have much influences on the adaptation field.

\section{Mislocalization during human outward adapta- tion}

The second goal of our study was to measure adaptation-induced mislocalization of visual targets within the outward adaptation field. Mislocalization was measured by asking subjects to localize a probe flashed more than $100 \mathrm{~ms}$ before saccade onset. This timing prevents interference from peri-saccadic compression (Ross et al., 1997; Awater et al., 2005; Bruno \& Morrone, 2007; Georg \& Lappe, 2009). We found that probe locations were systematically shifted in adaptation direction throughout the adaptation field.

We measured localization in three different conditions: target on, target off and fixation. Target on and target off condition were trials where subjects performed a saccade about $160 \mathrm{~ms}$ after a flash appeared at one of the 34 testing positions. The different trial types were included to investigate different sources of information that could be used for localization. The target on condition allows to use the post-saccadic target location as a reference for visual localization. Subjects may register the distance of the flash from the saccade target before saccade onset and then use the saccade target after saccade offset as a basis for their location report. Because the saccade target had jumped during the saccade the location report then would be shifted in the adaptation direction. The target off location does not allow to use the saccade target as a post-saccadic reference, because the target is extinguished during the saccade. Therefore, location reports can only be based on extraretinal information about the length of the saccade or post-saccadic eye position. Such signals are presumably available from an efference copy of the oculomotor command or from eye position sensitive neurons in the brain.

Like in inward adaptation (Collins et al., 2007) there was a clear difference in the mislocalization fields be- tween the target on and the target off conditions. This difference shows that the post-saccadic target is used in visual localization and that its shift is responsible for part of the mislocalization. However, mislocalization also occurred in the target off condition, showing that adaptation influences localization directly, not just via the step of the target. This is consistent with earlier studies that measured adaptation-induced mislocalization for adaption at a smaller range of positions (Awater et al., 2005; Bahcall \& Kowler, 1999; Bruno \& Morrone, 2007; Georg \& Lappe, 2009).

The spatial pattern of the directions of mislocalization differs between the target off and target on condition. Mislocalization in the target on condition appeared more uniform in direction and parallel to the adaptation direction than in the target off condition, in which the directions of mislocalization differed between positions and qualitatively showed a somewhat more radial arrangement with mislocalization being directed radially away from the fixation point. A similar difference between target on and target off mislocalization fields has been noted for inward adaptation. It can in part be explained by the influence of the target shift in the target on condition, which presumably imposes a uniform and parallel mislocalization at all locations. The correlation analysis showed a significant correlation between the mislocalization at a certain position and the adaptation-induced shift at that position in three out of four tests: the target on and target off conditions of the short saccade experiment and the target on condition of the long saccade experiment. These findings match with the report by Collins et al. (2007) for inward adaptation. The correlation between the mislocalization at a particular position and the adaptation at that position suggests that perceptual localization and adaptation are linked to each other, or that both adaptation and mislocalization independently increase with amplitude.

Mislocalization did not correlate with the adaptation of the standard saccade, i.e. the saccade that was actually performed in each localization trial. This shows that the mislocalization is not just the result of a change in saccade execution. Instead it must be related to the state of adaptation at every particular location in the field.

This finding is relevant for a potential contribution of the efference copy (Holst \& Mittelstaedt, 1950) of the adapted saccade to the adaptation-induced mislocalization. Collins et al. (2009) recently showed that saccadic suppression of displacement thresholds shifts with saccade adaptation, i.e. that even the saccade target is mislocalized after saccade adaptation (Collins, Rolfs, Deubel, \& Cavanagh, 2009) . Moreover, they showed that displacement judgments are independent of trial-to-trial variability in saccade amplitude. These findings are in line with the adaptation-induced mislocalization as the authors point out in their paper. As one possible explanation they discuss that the effer- 
ence copy is a faithful representation of the planned saccade but is unaware of saccade adaptation (Bahcall \& Kowler, 1999). The perceptual shift may then result from a mismatch between what the efference copy signal represents and the actual (adapted) landing position. However, in monkeys (Tanaka, 2003) as well as in humans (Panouilleres, Urquizar, Salemme, \& Pelisson, 2009) it has been demonstrated that in a double step paradigm where the first saccade is adapted the second saccade corrects for the gain change of the first saccade. These results suggest that the oculomotor signal has knowledge about the size of the adapatad saccade that it has executed. If the efference copy signal matches the actually executed saccade, localization based on this signal should be correct. As an alternative explanation Collins et al. (2007) discuss that the adaptation-induced mislocalization relies on a correct metric information about the adapted saccade, carried by the efference copy signal, but visual localization is directly linked to saccade targeting (Collins et al., 2007). This would imply that adaptation is attached to brain structures that support visual localization as well as saccade targeting. This explanation predicts that perceptual mislocalizations are not uniform throughout the visual field and that adaptation transfer is restricted to the vicinity of the saccade target, as has been found in the present study and in earlier studies (Awater et al., 2005; Collins et al., 2007; Bruno \& Morrone, 2007).

The finding that mislocalization is not simply related to saccade execution is corroborated by the results of the fixation condition. A significant, albeit small, mislocalization was observed in the short saccade experiment even when subjects continuously fixated and did not execute the saccade. Localization reports during fixation lay significantly more outward after adaptation than before adaptation. This is consistent with earlier reports of a difference in perceptual localization between outward and inward adaptation (Moidell \& Bedell, 1988) and recently reported transfers of outward adaptation to hand pointing (Hernandez et al., 2008) and perceptual localization (Zimmermann \& Lappe, 2010). The latter study addressed the adaptation induced mislocalization under fixation conditions in detail. It showed that during fixation and after outward adaptation flashed targets are mislocalized, but inward adaptation gave no mislocalization during fixation. However, the study also showed that the key factor was not the adaptation direction (inward or outward) but the size and persistence of the visual error. The stronger mislocalization during outward adaptation occured because outward adaptation is slower and less efficient, and thus has a large and persisting visual error even after many trials. When the error in the inward adaptation was made to persist, inward adaptation also began to show mislocalization during fixation. The short saccade experiment in the present study used a 14 deg saccade with a 6 deg outward step which is similar to the $13 \mathrm{deg}$ saccade with the $3 \mathrm{deg}$ outward step used by Zimmermann and Lappe (2010). Our finding of a significant mislocalization is thus consistent with their findings. The lack of effect in the long saccade experiment (20 deg amplitude, 6 deg step) may have to do with the larger amplitude, or with the smaller (in relation to the amplitude) step, or with the generally higher variance that our subjects showed in the localization data for the further eccentric positions. On the whole our fixation condition revealed a significant outward shift in the short saccade experiment, no such shift was found in saccadic inward adaptation (Collins et al., 2007) in accordance to the results of Zimmermann and Lappe (2010). Therefore this shift during fixation highlights certain differences between inward and outward adaptation processing which seem to rely on the size and persistence of the visual error during the course of adaptation. Consequently the size and persistence of the visual error may be controlled by slightly different adaptive mechanisms implicating that outward adaptation may use different stages of sensorimotor transformation than inward adaptation.

\section{References}

Alahyane, N., Devauchelle, A.-D., Salemme, R., \& Pelisson, D. (2008). Spatial transfer of adaptation of scanning voluntary saccades in humans. Neuroreport, 19(1), 37-41.

Alahyane, N., \& Pelisson, D. (2005). Long-lasting modifications of saccadic eye movements following adaptation induced in the double-step target paradigm. Learning $\mathcal{E}$ Memory, 12(4), 433-443.

Albano, J. E. (1996). Adaptive changes in saccade amplitude: oculocentric or orbitocentric mapping? Vision Research, 36(14), 2087-2098.

Albano, J. E., \& King, W. M. (1989). Rapid adaptation of saccadic amplitude in humans and monkeys. Investigative Ophthalmology \& Visual Science, 30(8), 1883-1893.

Awater, H., Burr, D., Lappe, M., Morrone, M. C., \& Goldberg, M. E. (2005). Effect of saccadic adaptation on localization of visual targets. Journal of Neurophysiology, 93(6), 36053614.

Bahcall, D. O., \& Kowler, E. (1999). Illusory shifts in visual direction accompany adaptation of saccadic eye movements. Nature, 400(6747), 864-866.

Bischof, N., \& Kramer, E. (1968). Untersuchungen und Ueberlegungen zur Richtungswahrnehmung bei willkuerlichen sakkadischen Augenbewegungen. Psychologische Forschung, 32, 185-218.

Bruno, A., \& Morrone, M. C. (2007). Influence of saccadic adaptation on spatial localization: Comparison of verbal and pointing reports. Journal of Vision, 7(5)(16), 1-12.

Catz, N., Dicke, P. W., \& Thier, P. (2008). Cerebellardependent motor learning is based on pruning a purkinje cell population response. Proceedings of the National Academy of Sciences of the United States of America, 105(20), $7309-7314$

Cecala, A. L., \& Freedman, E. G. (2009). Head-Unrestrained Gaze Adaptation in the Rhesus Macaque. Journal of Neurophysiology, 101(1), 164-183.

Chen-Harris, H., Joiner, W. M., Ethier, V., Zee, D. S., \& Shad- 
mehr, R. (2008). Adaptive control of saccades via internal feedback. Journal of Neuroscience, 28(11), 2804-2813.

Collins, T., \& Dore-Mazars, K. (2006). Eye movement signals influence perception: Evidence from the adaptation of reactive and volitional saccades. Vision Research, 46(21), 3659 $-3673$.

Collins, T., Dore-Mazars, K., \& Lappe, M. (2007). Motor space structures perceptual space: Evidence from human saccadic adaptation. Brain Research, 1172, 32-39.

Collins, T., Rolfs, M., Deubel, H., \& Cavanagh, P. (2009). Postsaccadic location judgments reveal remapping of saccade targets to non-foveal locations. Journal of Vision, 9(5).

Cotti, J., Guillaume, A., Alahyane, N., Pelisson, D., \& Vercher, J.-L. (2007). Adaptation of Voluntary Saccades, But Not of Reactive Saccades, Transfers to Hand Pointing Movements. Journal of Neurophysiology, 98(2), 602-612.

Deubel, H. (1987). Adaptivity of gain and direction in oblique saccades. In J. K. O'Regan \& A. Levy-Schoen (Eds.), Eye movements: From physiology to cognition (p. 181-190). New York: Elsevier/North-Holland.

Deubel, H. (1991). Adaptive control of saccade metrics. In G. Obrecht \& G. Stark (Eds.), Presbyopia research (p. 93-100). New York: Plenum Press.

Deubel, H. (1995). Separate adaptive mechanisms for the control of reactive and volitional saccadic eye movements. Vision Research, 35, 3529-3540(12).

Deubel, H., Wolf, W., \& Hauske, G. (1986). Adaptive gain control of saccadic eye movements. Human Neurobiology, $5(4), 245-253$.

Ethier, V., Zee, D. S., \& Shadmehr, R. (2008). Changes in control of saccades during gain adaptation. Journal of Neuroscience, 28(51), 13929-13937.

Frens, M. A., \& Van Opstal, A. J. (1994). Transfer of short-term adaptation in human saccadic eye movements. Experimental Brain Research, 100, 293-306.

Fujita, M., Amagai, A., Minakawa, F., \& Aoki, M. (2002). Selective and delay adaptation of human saccades. Cognitive Brain Research, 13(1), 41-52.

Georg, K., \& Lappe, M. (2009). Effects of saccadic adaption on visual localization before and during saccades. Experimental Brain Research, 129, 9-23.

Golla, H., Tzidris, K., Haarmeier, T., Catz, N., Barash, S., \& Thier, P. (2008). Reduced saccadic resilience and impaired saccadic adaptation due to cerebellar disease. European Journal of Neuroscience, 27(1), 132-144.

Hernandez, T. D., Levitan, C. A., Banks, M. S., \& Schor, C. M. (2008). How does saccade adaptation affect visual perception? Journal of Vision, 8 (8)(3), 1-16.

Holst, E., \& Mittelstaedt, H. (1950). Das reafferenzprinzip. Naturwissenschaften, 37, 464-476.

Honda, H. (1989). Perceptual localization of visual stimuli flashed during saccades. Perception \& Psychophysics, 45(2), 162-174.

Honda, H. (1991). The time courses of visual mislocalization and of extraretinal eye position signals at the time of vertical saccades. Vision Research, 31(11), 1915 - 1921.

Honda, H. (1993). Saccade-contingent displacement of apparent position of visual stimuli flashed on a dimly illuminated structure background. Vision Research, 33(5/6), 709-716.

Hopp, J. J., \& Fuchs, A. F. (2004). The characteristics and neuronal substrate of saccadic eye movement plasticity. Progress in Neurobiology, 72(1), 27-53.
Kaiser, M., \& Lappe, M. (2004). Perisaccadic mislocalization orthogonal to saccade direction. Neuron, 41(2), 293 - 300.

Matin, L., Pearce, E., \& Pola, J. (1970). Visual perception of direction when voluntary saccades occur: II. relation of visual direction of a fixation target extinguished before saccade to a subsequent test flash presented before the saccade. Perception $\mathcal{E}$ Psychophysics, 8(1), 9-14.

McLaughlin, S. (1967). Parametric adjustment in saccadic eye movements. Perception E Psychophysics., 2, 359-362.

Miller, J. M., Anstis, T., \& Templeton, W. B. (1981). Saccadic plasticity: Parametric adaptive control by retinal feedback. Journal of Experimental Psychology: Human Perception \& Performance, $7(2), 356-366$.

Moidell, B. G., \& Bedell, H. E. (1988). Changes in oculocentric visual direction induced by the recalibration of saccades. Vision Research, 28(2), 329-336.

Morrone, M. C., Ross, J., \& Burr, D. C. (1997). Apparent Position of Visual Targets during Real and Simulated Saccadic Eye Movements. Journal of Neuroscience, 17(20), 7941-7953.

Noto, C. T., \& Robinson, F. R. (2001). Visual error is the stimulus for saccade gain adaptation. Cognitive Brain Research, 12(2), 301-305.

Noto, C. T., Watanabe, S., \& Fuchs, A. F. (1999). Characteristics of simian adaptation fields produced by behavioral changes in saccade size and direction. Journal of Neurophysiology, 81(6), 2789-2813.

Optican, L. M., \& Robinson, D. A. (1980). Cerebellardependent adaptive control of primate saccadic system. Journal of Neurophysiology, 44(6), 1058-1076.

Panouilleres, M., Urquizar, C., Salemme, R., \& Pelisson, D. (2009). Non-visual signals used for programming sequence of saccades originate downstream from the locus of saccadic adaptation. (Talk at 15th European Conference On Eye Movements, Southampton 2009)

Panouilleres, M., Weiss, T., Urquizar, C., Salemme, R., Munoz, D. P., \& Pelisson, D. (2008). Behavioural evidence of separate adaptation mechanisms controlling saccade amplitude lengthening and shortening. Journal of Neurophysiology, 101(3), 1550 -1559.

Pelisson, D., Alahyane, N., Panouilleres, M., \& Tilikete, C. (2010). Sensorimotor adaptation of saccadic eye movements. Neuroscience \& Biobehavioral Reviews, 34(8), 11031120.

Pierrot-Deseilligny, C. (1991). Cortical control of saccades. Neuro-Ophthalmology, 11(2), 63-75.

Robinson, F. R., Noto, C. T., \& Bevans, S. E. (2003). Effect of visual error size on saccade adaption in monkey. Journal of Neuroscience, 90, 1235-1244.

Ross, J., Morrone, M. C., \& Burr, D. C. (1997). Compression of visual space before saccades. Nature, 386, 598-601.

Scudder, C. A., Batourina, E. Y., \& Tunder, G. S. (1998). Comparison of Two Methods of Producing Adaptation of Saccade Size and Implications for the Site of Plasticity. Journal of Neurophysiology, 79(2), 704-715.

Semmlow, J. L., Gauthier, G. M., \& Vercher, J. L. (1989). Mechanisms of short-term saccadic adaptation. Journal of Experimental Psychology: Human Perception \& Performance, 15(2), 249-258.

Shafer, J. L., Noto, C. T., \& Fuchs, A. F. (2000). Temporal Characteristics of Error Signals Driving Saccadic Gain Adaptation in the Macaque Monkey. Journal of Neurophysiology, 84(1), 88-95.

Straube, A., \& Deubel, H. (1995). Rapid gain adaptation af- 
fects the dynamics of saccadic eye movements in humans. Vision Research, 35(23-24), 3451-3458.

Straube, A., Fuchs, A. F., Usher, S., \& Robinson, F. R. (1997). Characteristics of Saccadic Gain Adaptation in Rhesus Macaques. Journal of Neurophysiology, 77(2), 874-895.

Tanaka, M. (2003). Contribution of signals downstream from adaptation to saccade programming. Journal of Neurophysiology, 90, 2080-2086.

Wallman, J., \& Fuchs, A. F. (1998). Saccadic gain modification: visual error drives motor adaptation. Journal of Neurophysiology, 80(5), 2405-2416.

Watanabe, S., Noto, C. T., \& Fuchs, A. F. (2000). Flexibility of saccade adaptation in the monkey: different gain states for saccades in the same direction. Experimental Brain Research, 130(2), 169-176.

Xu-Wilson, M., Chen-Harris, H., Zee, D. S., \& Shadmehr, R. (2009). Cerebellar contributions to adaptive control of saccades in humans. Journal of Neuroscience, 29(41), 1293012939.

Zimmermann, E., \& Lappe, M. (2009). Mislocalization of Flashed and Stationary Visual Stimuli after Adaptation of Reactive and Scanning Saccades. Journal of Neuroscience, 29(35), 11055-11064.

Zimmermann, E., \& Lappe, M. (2010). Motor signals in visual localization. Journal of Vision, 10(6:2), 1-11. 